\title{
On the nature of nearby GRB/SN host galaxies *
}

\author{
J. Sollerman, ${ }^{\mathrm{a}, \mathrm{b}, *}$ G. Östlin, ${ }^{\mathrm{a}}$ J. P. U. Fynbo, ${ }^{\mathrm{b}}$ J. Hjorth, ${ }^{\mathrm{b}}$ \\ A. Fruchter, ${ }^{\mathrm{c}}$ K. Pedersen ${ }^{\mathrm{b}}$ \\ ${ }^{a}$ Department of Astronomy, Stockholm University, AlbaNova, 10691 Stockholm, Sweden \\ ${ }^{\mathrm{b}}$ DARK, Niels Bohr Institute, University of Copenhagen, Juliane Maries Vej 30, DK-2100 \\ Copenhagen $\emptyset$, Denmark \\ ${ }^{\mathrm{c}}$ Space Telescope Science Institute, 3700 San Martin Drive, Baltimore, MD 21218, USA
}

\begin{abstract}
We present and discuss optical diagnostics of the low redshift $(z<0.2)$ galaxies that are known to have hosted supernovae associated with $\gamma$-ray bursts (GRBs). The three galaxies are all actively starforming sub-luminous $\left(L<L^{\star}\right)$ galaxies with relatively low metallicities $\left(Z \lesssim Z_{\odot}\right)$. We find no evidence for substantial internal extinction within any of the galaxies. We derive star formation rates (SFR) based on $\mathrm{H} \alpha$ luminosities, as well as specific star formation rates (SSFR, star formation rate per unit luminosity). For GRB 980425 (SN 1998bw) we use photometry of the supernova environment to estimate the mass of the progentitor to $\gtrsim 30 M_{\odot}$. These three host galaxies have global properties (luminosities, SFR, SSFR, metallicity, colour, reddening) that resemble those of more distant GRB host galaxies. We also compare the host galaxies with a sample of Blue Compact Galaxies (BCGs) in the local universe, and show that these samples have similar properties.
\end{abstract}

Key words:

gamma rays bursts, supernovae SN 1998bw, SN 2003dh, SN 20031w

\section{Introduction}

It is now well established that long-duration $\gamma$-ray bursts (GRBs) coincide with the explosions of certain massive stars, i.e., with a subset of very energetic core-

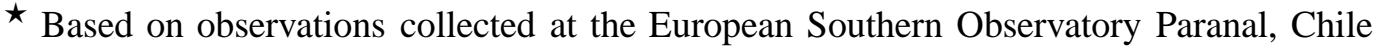
(ESO Programmes 63.H-0649 and 271.D-5006). Also partly based on programmes 70.D0087 and 165.H-0464. Partly based on HST observations obtained under programme 9405.

* J. Sollerman, email: jesper@astro.su.se
} 
collapse supernovae $(\mathrm{SNe})$. In particular, three such cases must be regarded as secure.

The first was the association of SN 1998bw with GRB 980425 (Galama et al., 1998). This connection was, however, quite debated until an unambiguous association was revealed between GRB 030329 and SN 2003dh (Hiorth et al., 2003; Stanek et al., 2003). SN 2003dh also showed properties almost identical to that of SN 1998bw. A third event has now filled in the picture with the spectroscopically confirmed association between GRB 031203 and SN 20031w (Thomsen et al., 2004; Malesani et al., 2004).

Because the detection of GRBs in $\gamma$-rays is unaffected by intervening gas and dust, they provide a powerful and possibly unbiased tracer of star-formation in the high- $z$ universe. This highlights the importance of studies of GRB host galaxies. Previous studies indicate that the majority of GRB host galaxies are blue and sub-luminous (e.g., Fruchter et al., 1999; Le Floc'h et al., 2003; Jakobsson et al., 2005).

Based on these properties of GRB host galaxies, Watson et al. (2004) discussed whether a sizable portion of global star-formation occurs in small and rather unobscured, modestly star-forming galaxies that are too faint to appear in other surveys of star-formation activity, or whether GRBs trace only a fraction of the star-forming population, for example due to metallicity effects (e.g., Fynbo et al., 2003).

The most nearby GRB host galaxies, and the ones that are undoubtedly directly connected to the deaths of massive stars, allow for a more detailed analysis. Watson et al. (2004) used X-ray observations to constrain the star-formation rates in the host galaxies of the three SN-GRB associations mentioned above. In the present study, we will instead use optical observations to address the same questions. In Sect. 2 we present the data on the hosts and shortly describe the performed data reductions. In Sect. 3 we characterize the host galaxies in terms of emission line fluxes, constraints on metallicities and extinctions, star formation rates, and physical dimensions. In Sect. 4 we discuss and compare the obtained results of the nearby GRB host galaxies with a population of blue compact galaxies. Our findings are summarized in Sect. 5

A cosmology where $H_{0}=70 \mathrm{~km} \mathrm{~s}^{-1} \mathrm{Mpc}^{-1}, \Omega_{\Lambda}=0.7$ and $\Omega_{\mathrm{m}}=0.3$ is assumed throughout. The redshifts of the three host galaxies; SN 1998bw at $z=0.0085, \mathrm{SN}$ 2003lw at 0.1055 and SN $2003 \mathrm{dh}$ at 0.1685 then correspond to luminosity distances of 37, 487 and $810 \mathrm{Mpc}$, respectively. 


\section{Observations and data reduction}

\subsection{Spectroscopic data for the GRB 980425 host}

The host of GRB 980425 and SN 1998bw, ESO 184-G82, was extensively observed as part of several monitoring programmes for SN 1998bw. The evolution of the SN is described in detail elsewhere (Sollerman et al., 2000; Patat et al., 2001; Sollerman et al., 2002). Here we will make use of some of these observations to characterize the host galaxy. We have used the late spectroscopy from 13 June 1999 obtained at the ESO Very Large Telescope (VLT), using the Focal Reducer and low dispersion Spectrograph (FORS1) instrument. These data are presented in Sollerman et al. (2000) and the spectra clearly reveal a number of narrow emission lines from the underlying host galaxy. The spectra were obtained using the $300 \mathrm{~V}$ and $300 \mathrm{I}$ grisms and a 1 .'0 wide slit and cover the wavelength region $\sim 3700-9700 \AA$. The spectra were reduced in a standard way, including bias subtraction, flat fielding, and wavelength calibration using spectra of a Helium-Argon lamp. Flux calibration was done relative to the spectrophotometric standard star LTT 7379 (Hamuy et al., 1994). We did not only use the reduction of Sollerman et al. (2000), which aimed at reducing the presence of host contamination in the SN spectrum. Instead we re-reduced the data to better reveal the underlying emission line region. We also extracted spectra of two other H II regions in the part of the host covered by the slit (see Fig. 1).

The absolute flux calibration of the combined spectrum was obtained relative to our well-calibrated broad-band SN photometry. However, since the slit does not cover the entire galaxy, we can not derive the total emission line flux and hence not estimate the global star formation rate. This is instead done from narrow band imaging (Sect. 2.2).

\subsection{Narrow band imaging for the GRB 980425 host}

For ESO 184-G82 we have also retrieved narrow band imaging data from the ESO archive. In particular, we wanted to use the $\mathrm{H} \alpha$ imaging to estimate the global star formation rate of the galaxy. These observations were performed on 3 August 2000 with the FORS1 instrument on the VLT. Three 5 minute exposures were obtained in a narrow filter centered on $\mathrm{H} \alpha$ at the host galaxy redshift. Another five minute exposure was obtained in a zero-velocity narrow $\mathrm{H} \alpha$ filter, to allow for continuum subtraction. The data were reduced in a standard way and the continuum flux-calibration was performed relative to a photometric standard star (PG 1657, Landolt, 1992) observed immediately before the galaxy at a similar airmass. The broad-band zero-points obtained from this standard star indicates that the night was photometric. 


\subsection{Broad band imaging for the GRB 980425 host}

We have re-analysed the late time BVRI imaging of the host galaxy of SN 1998bw, which were originally used as templates for the $\mathrm{SN}$ template subtraction photometry in Sollerman et al. (2002). These are very deep images obtained with FORS1 at a time when the SN flux was negligible. We use it here to construct a spectral energy distribution (SED) for the galaxy. The photometry was calibrated against local

photometric standard stars (Sollerman et al., 2002). The photometry for the entire galaxy is consistent with the photographic ESO-Uppsala catalogue (Lauberts \& Valentijn, 1989).

We also did photometry of the small H II region that contained the SN (see e.g., Fynbo et al., 2000; Sollerman et al., 2002). The magnitude was measured in an aperture with a diameter of 9 pixels (1".8), and the local background was subtracted.

\subsection{Spectroscopic data for the GRB 030329 host}

Spectroscopy of the host of GRB 030329 was obtained on 19 June 2003 with the FORS2 instrument on the VLT. As outlined by Gorosabel et al. (2005) these observations were conducted with the $300 \mathrm{~V}$ grism and order sorting filter GG375, which efficiently covers the wavelength range from $\sim 3800-8800 \AA$. The 1 .'3 wide slit was used and the seeing during the observations was below $0{ }^{\prime} 6$. Given the small size of the object (see Fig. 1), the bulk of the flux from the host galaxy should be included in the slit. Also note that we used a position angle of 123.6 degrees East of North, which is well aligned with the orientation of the host galaxy (Fig. 1).

The spectra were reduced in the standard way. Flux calibration was done relative to the spectrophotometric standard star Feige 67 (Oke, 1990). The absolute flux calibration of the combined spectrum was again obtained relative to broad-band photometry. A figure showing this spectrum is shown by Gorosabel et al. (2005).

\subsection{High resolution spectra for the GRB 030329 host}

We retrieved early high-resolution spectra of GRB 030329 from the ESO archive, mainly with the aim of searching for the absorption lines of Na I D and hence constrain the amount of extinction along the line-of-sight to the burst. These lines are often used in SN studies for this purpose (see e.g., Sollerman et al., 2005). The observations were obtained 16 hours after the burst on 2003 March 30 with the Ultraviolet and Visual Echelle Spectrograph (UVES) on the VLT (Greiner et al., 2003). We reduced the spectra using the UVES-pipeline as implemented in MIDAS. This reduction package allows for bias subtraction and flat-fielding of the data using 
calibration frames obtained during day time. Very accurate wavelength calibration was secured by comparison to ThAr arc lamp spectra. From the reduced spectrum we also confirm the redshift reported by Greiner et al. (2003) (see also Stanek et al., 2003; Hiorth et al., 2003), $z=0.1685$. The [O III] $\lambda 5006.9$ line was measured at $\lambda$ 5850.69, which yields $\mathrm{z}=0.168525$, and $\mathrm{H} \alpha \lambda 6562.8$ was measured at $\lambda 7668.82$ which gives $\mathrm{z}=0.168528$. The measured wavelengths are corrected for the barycentric velocity.

\section{Results}

\subsection{Emission line fluxes}

The measured emission line fluxes from the host galaxies of GRB 980425 and GRB 030329 are given in Table 1. In that table we also provide line fluxes for the host galaxy of GRB 031203, from the thorough analysis presented by Prochaska et al. (2004).

We note that for GRB 030329 the main difference between the results presented by Gorosabel et al. (2005) and those by Hiorth et al. (2003) is the absence of significant [N II] emission. The detection of this emission line by Hiorth et al. (2003) in the spectrum from 1 May 2003 appears to have been spurious. It is not seen in any of the other spectra published by Hiorth et al. (2003) and the lack of [N II] emission is also noted by Matheson et al. (2003).

\subsection{Metallicities}

From the measured emission line fluxes we have made an attempt to constrain the metallicities of the three host galaxies. We followed the methods outlined by

Lee et al. (2003) and by Kewley \& Dopita (2002) to derive metallicity estimates from the strong emission lines. These estimates are based on the R23 technique which is an empirical relation between the oxygen abundance and the intensity ratio of the strong oxygen emission lines ([O II] $3727 \AA$, and [O III] 4959,5007 $\AA$ ) to $\mathrm{H} \beta$. This relationship is, however, not unique and therefore provides two possible solutions (lower and upper branch) for the nebular oxygen abundance. This degeneracy may be resolved by including other lines such as [N II] $6584 \AA$ A.

\subsubsection{The GRB 030329 host}

Using the prescription of Lee et al. (2003) for the emission line strengths of oxygen measured for the host of GRB 030329 we can derive a metallicity of either $\sim 7.9$ 
(lower branch) or $~ 8.6$ (upper branch). The lower branch value would suggest a metallicity significantly below solar which would also be consistent with the luminosity of the galaxy (see, e.g., Fig. 6 in Lee et al., 2003) (or Fig. 4 in Lee et al., 2004). However, based on the emission lines alone we can not exclude a metallicity closer to solar $\left(12+\log (\mathrm{O} / \mathrm{H})_{\odot}=8.7\right.$, Allende Prieto et al., 2001).

Despite the depth of the VLT/FORS spectrum, [O III] $4363 \AA$ and [N II] $6584 \AA$ were not detected. Using the various diagnostic tools summarized by Kewley \& Dopita (2002) we did not manage to break the degeneracy of the oxygen abundance solution based on the R23 ratio. In fact, using our upper limit on [N II] together with the [O II], $\mathrm{H} \alpha$, and [O III] fluxes only gives weak constraints for most published diagnostic diagrams (Edmunds \& Pagel, 1984; Kewley \& Dopita, 2002), i.e., $12+\log (\mathrm{O} / \mathrm{H})<8.6$. Also our [S II] detection gives only weak constraints when compared to [N II] and $\mathrm{H} \alpha$. Hence we could not constrain the oxygen abundance of the host of GRB 030329 using the emission line fluxes, but it appears to be subsolar.

\subsubsection{The GRB 980425 host}

For the host galaxy of GRB 980425 we have performed a similar analysis based on the emission line fluxes. We caution that the absolute fluxing in the bluest part of the spectrum, where [O II] is located, may be affected by systematic uncertainties in the flux-calibration. This, as well as uncertainties in the extinction corrections may affect the metallicity estimates, but does not alter the overall results.

We extracted spectra from three spatial locations within the host galaxy (Fig. 1). The metallicities were then derived using the recipe in Kewley \& Dopita (2002). In this case, we have enough information to support the upper branch of the R23 diagnostic, and find the metallicities shown in Table 2. Thus, this galaxy does not have a very low metallicity. There may be a slight variation in the metallicity across the galaxy, but given the uncertainties we do not regard this as a significant result.

We note that the emission lines of [Ne III] are stronger at the site of the burst. As argued by Bloom et al. (1998) this is indicative of a high degree of ionization, suggesting a substantial population of young and massive stars. Such an interpretation is also supported by our modeling of the broad-band spectral energy distribution (Sect. 3.5).

\subsection{Extinction}

The collected dataset allows some estimates of the extinction in the hosts. The extinctions are also needed for estimating the star formation rates below. 
For the GRB 030329 host galaxy there are several estimates of the extinction available. First, the photometric spectral energy distribution of the host as fitted by Gorosabel et al. (2005) favours a starburst galaxy with an intrinsic $E(B-V) \sim$ 0.2 mag. The Galactic extinction in this direction is estimated to be $E(B-V)=$ 0.025 mag (Schlegel et al., 1998). Matheson et al. (2003) also argued for a low extinction. They based their arguments on the Balmer decrement and also made an estimate based on the assumed power-law properties of the afterglow emission. The latter method gave a limit on the extinction towards the burst of $E(B-V)=$ $0.04 \pm 0.08$, implying that there is no evidence for extragalactic dust along the line of sight between us and GRB 030329.

The high-resolution spectroscopy of the afterglow of GRB 030329 discussed above (Sect. 2.5) also speaks in favor of a very low amount of dust towards the GRB. At the position of the Galactic sodium lines we do detect a weak line. The $\lambda 5890$ component has an equivalent width $(\mathrm{EW})$ of $\sim 40 \mathrm{m \AA}$. This small EW is fully consistent with the low amount of Galactic extinction in this direction (e.g., Hobbs, 1974; Sollerman et al., 2005). At the position of the Na I D lines at the redshift of GRB 030329 we detect no significant absorption. Any such absorption must be at least four times weaker than the Galactic component. Although the degree of ionization could be different in the GRB 030329 host and the Galaxy, this at least points to a very small amount of reddening along the line of sight to GRB 030329.

We must remember, however, that the line-of-sight towards the GRB need not be representative for the entire host galaxy. It may even be that dust is destroyed by the burst itself (e.g., Waxman \& Draine, 2000; Galama \& Wijers, 2001; Fruchter et al., 2001).

For the GRB 980425 host galaxy we have limited information on the extinction. The Galactic extinction in this direction is estimated to be $E(B-V)=0.059 \mathrm{mag}$ (Schlegel et al., 1998). According to Patat et al. (2001) there was no sign of Na I D absorption in high-resolution spectra obtained at the SN maximum. These authors used this finding to argue for an extinction of $E(B-V)<0.065$ mag towards the SN, i.e., a again very small amount of reddening. Also the Balmer decrement from the spectra indicate a low reddening, which is also consistent with our SED modeling (Sect. 3.5.1).

\subsection{Star Formation Rates}

There are several ways to estimate star formation rates (SFR) in galaxies. One option is simply to use the emission line luminosities of $\mathrm{H} \alpha$ or [O II] and follow the prescription by Kennicutt (1998).

For the GRB 980425 host galaxy we have used narrow band imaging to estimate the flux in $\mathrm{H} \alpha$, since this galaxy is much larger than the extent of our spectroscopic 
slit.

The final continuum subtracted image is shown in Fig. 1. The host galaxy is clearly visible. We also note that no other source was detected in the field of view. From this image we derived an integrated $\mathrm{H} \alpha$ flux of $2.6 \times 10^{-13} \mathrm{erg} \mathrm{s}^{-1} \mathrm{~cm}^{-2}$. The integrated EW is $60.6 \AA$. If we correct these for a $10 \%$ contribution from the [N II] emission line within the filter band (as estimated from the spectroscopy), and for a Galactic extinction of $E(B-V)=0.059 \mathrm{mag}$ we get for the distance of GRB $980425 \mathrm{a}$ luminosity of $\mathrm{L}_{\mathrm{H} \alpha}=4.4 \times 10^{40} \mathrm{erg} \mathrm{s}^{-1}$. The corrected $\mathrm{EW}$ is $54.5 \AA$.

We thus derive a global SFR of $\sim 0.35 \mathrm{M}_{\odot} \mathrm{yr}^{-1}$ while the bright $\mathrm{H}$ II region northwest of the explosion site of SN 1998BW has $\sim 0.11 \mathrm{M}_{\odot} \mathrm{yr}^{-1}$. These estimates have also been corrected for Galactic extinction and for a $10 \%$ contamination of [N II] emission inside the narrow band filter.

For the GRB 030329 host we obtain the following integrated SFR;

$\mathrm{SFR}_{\mathrm{H} \alpha}=0.22 \mathrm{M}_{\odot} \mathrm{yr}^{-1}$

$\mathrm{SFR}_{[\mathrm{O} \text { II }]}=0.22 \mathrm{M}_{\odot} \mathrm{yr}^{-1}$

We note that these values are consistent with the values reported by Hiorth et al. (2003). The $\mathrm{H} \alpha$ line sits close to a telluric absorption line and was not used by Gorosabel et al. (2005) to estimate the SFR. These values are for a Galactic extinction only. An extinction as suggested by Gorosabel et al. (2005), $E(B-V)=0.2$ would alter these numbers to

$\mathrm{SFR}_{\mathrm{H} \alpha}=0.32 \mathrm{M}_{\odot} \mathrm{yr}^{-1}$

$\mathrm{SFR}_{[\mathrm{O} \text { II }]}=0.48 \mathrm{M}_{\odot} \mathrm{yr}^{-1}$

This of course applies only to the part of the galaxy included in the slit - although we argue that this was actually the major part.

These estimates are similar to the SFR reported by Matheson et al. (2003), $0.5 \mathrm{M}_{\odot} \mathrm{yr}^{-1}$, although the agreement appears to be somewhat accidental. We were unable to reproduce their high $\mathrm{L}_{\mathrm{H} \alpha}$ also from their publicly available spectra, but on the other hand they assume a much lower extinction correction.

The SFR estimated from the optical emission lines must be regarded as lower limits, since there may also exists an extinguished star-forming population, as in many other starburts. For example, the host of GRB 000210 revealed a SFR $2-3 \mathrm{M}_{\odot} \mathrm{yr}^{-1}$ from the optical emission lines (Gorosabel et al., 2003), while a tentative sub-mm detection implies a star formation of several hundred solar-masses per year (Berger et al., 2003).

In the case of the SN/GRB hosts we also have upper limits on the star formation 
as obtained from the unbiased X-ray view exploited by Watson et al. (2004). For the GRB 980425 host they find that the X-ray flux within the optical extent of this galaxy is entirely dominated by two point sources $\sim 1.5^{\prime \prime}$ apart, one of which is coincident with the radio position of SN 1998bw and is almost certainly associated with it (Watson et al., 2004; Kouveliotou et al., 2004).

From the X-ray emission they estimated a total SFR of $2.8 \pm 1.9 \mathrm{M}_{\odot} \mathrm{yr}^{-1}$. Together with our estimate of $0.35 \mathrm{M}_{\odot} \mathrm{yr}^{-1}$ the range is therefore quite well constrained for this host galaxy.

For GRB 030329 we have estimated SFR $0.4 \mathrm{M}_{\odot} \mathrm{yr}^{-1}$ while Watson et al. (2004) estimate a SFR of massive stars $\left(\mathrm{M} \gtrsim 5 M_{\odot}\right.$, see Watson et al. 2004; Grimm et al. 2003 ) of less than $31 \pm 13 \mathrm{M}_{\odot} \mathrm{yr}^{-1}$. Using a salpeter IMF (Salpeter, 1955; Persic et al., 2004; Watson et al., 2004) this corresponds to a total SFR of $<200 \pm 80 \mathrm{M}_{\odot} \mathrm{yr}^{-1}$.

\subsection{Luminosities, Physical sizes and Galaxy types.}

\subsubsection{The GRB 980425 host}

The host of GRB 980425, ESO 184-G82, is nearby enough to be well resolved with ground based telescopes (Fig. 1) and appears to be a late type spiral with a bar (SBc). The beautiful HST image displayed by Fynbo et al. (2000) shows the optical appearance of the galaxy to be dominated by a large number of high surface brightness starforming regions, especially in the southern spiral arm where the GRB/SN occurred.

The total BVRI magnitudes we obtained from our VLT images down to an isophote of 25 magnitudes $\operatorname{arcsec}^{-2}$ are reported in Table 3. For the adopted distance this gives an absolute magnitude of $\mathrm{M}_{B}=-17.65$. Adopting $\mathrm{M}_{B}^{\star}=-21$ we thus find that this galaxy has $L=0.05 L^{\star}$. We can use this and the above derived SFR to also get the specific star formation rate (SSFR). For GRB 980425 we thus derive a $\mathrm{SSFR} \sim 7 \mathrm{M}_{\odot} \mathrm{yr}^{-1}\left(\mathrm{~L} / \mathrm{L}^{\star}\right)^{-1}$.

The major axis diameter of the galaxy is 67 arcseconds at the $\mathrm{B}=26.5 \mathrm{mag} \operatorname{arcsec}^{-2}$ isophote, corrected for foreground Milky way extinction, i.e. this is the Holmberg diameter. The minor axis is 57 arcseconds. At a distance of $37 \mathrm{Mpc}$ this corresponds to a physical size of $12 \times 10 \mathrm{kpc}$.

Comparing our broadband magnitudes to empirical galaxy colours (e.g., Coleman et al., 1980) we see that the host appears to be a typical, subluminous late type spiral. To quantitatively compare the colors of the host galaxy we calculated a set of models using the code PEGASE.2 (Fioc \& Rocca-Volmerange, 1997, 1999) which includes both stellar and nebular emission. This was done using the actual filter profiles and CCD sensitivity for FORS1, and all models assume a Salpeter IMF in the mass 
interval 0.1-120 $\mathrm{M}_{\odot}$. Using a range of different metallicities and star formation timescales we could then compare these models to our data using least-square fitting.

The entire galaxy is well fit with a continuous star formation history. Both an efolding time of $3 \mathrm{Gyr}$ and $15 \mathrm{Gyr}$ give good agreements without additional extinction. The results are not very sensitive to metallicity. Independently, the $\mathrm{H} \alpha \mathrm{EW}$ is well fit by such a scenario. Both these scenarios are also able to reproduce the integrated luminosity of the galaxy given that the current SFR has operated for 5-7 Gyrs. The galaxy can thus not be regarded as a starburst galaxy.

\subsubsection{The GRB 980425 progenitor mass}

For the local SN/GRB environment we have conducted a similar exercise. The photometry is presented in Table 3 and the results of the modeling are quantified and summarized in Table 4. We assumed an instantaneous burst and the same IMF parameters as above. The best fitting age is for each metallicity a well defined minimum, where a change in age as small as \pm 1 Myr typically increases the RMS deviation with a factor two or more. For all models, the best-fitting internal reddening is found to be $E(B-V)=0.05$. As is illustrated in Table 4, a change in $E(B-V)$ of \pm 0.05 , leads to an increased RMS with typically 50 to $100 \%$, but does not affect the best fitting age. Hence, the uncertainties on the derived ages are very small $(\sim 1$ Myr) in a statistical sense.

If we take the best fitting age as the lifetime of the supernova progenitor we can estimate its mass from comparison with stellar tracks (Bressan et al., 1993; Fagotto et al., 1994; Meynet et al., 1994). This gives a ZAMS mass of $30 \pm 5 \mathrm{M}_{\odot}$, in accordance with most models for Type Ic SNe in general, and for collapsars in particular.

\subsubsection{The GRB 030329 host}

For GRB 030329 the magnitudes reported by Gorosabel et al. (2005) imply $L=$ $0.016 L^{\star}$. With our measured (extinction corrected) SFR this gives a SSFR of $25 \mathrm{M}_{\odot} \mathrm{yr}^{-1}\left(\mathrm{~L} / \mathrm{L}^{\star}\right)^{-1}$. As noted by Gorosabel et al. (2005) this is a very high SSFR compared to most galaxies in the Hubble Deep Field.

To constrain the physical size of this host galaxy we used images obtained with the ACS onboard the Hubble Space Telescope. We measured the extent of the host on ACS images obtained on 2004 May 24, i.e., 422 days after the GRB and thus long after the afterglow contribution had vanished.

The FWHM of the host is about $530 \times 930$ pc in the F435W filter image. The F606W and F814W filters show a slightly larger FWHM of $\sim 620 \times 1030 \mathrm{pc}$. 
This is similar to the radius estimated by fitting a Sersic model to the surface brightness profile (e.g., Warren et al., 2001), which gave a radius of 0.26 arcseconds corresponding to $750 \mathrm{pc}$ for the F606W filter. We also estimated the Holmberg diameter to compare directly with the estimate of the GRB 980425 host galaxy. This gave instead 1.'4, corresponding to $3.9 \mathrm{kpc}$.

The main conclusion of this exercise is that the host galaxy of GRB 030329 is a very compact galaxy, with both an absolute magnitude and extension similar to that of the SMC. Furthermore, our UVES spectroscopy (Sect. 2.5) resolved the H $\alpha$ line with a FWHM of $\sim 55 \mathrm{~km} \mathrm{~s}^{-1}$, which for a radius of $0.75 \mathrm{kpc}$ corresponds to a dynamical mass of $\sim 5 \times 10^{8} \mathrm{M}_{\odot}$ (e.g., Östlin et al., 2001). This may be an underestimate if the $\mathrm{H} \alpha$ flux is dominated by a central burst and do not trace the full potential well. The dynamical mass is similar to the mass of SMC. In Fig. 1 we show the host galaxies of GRBs 030329 and 980425 on the same physical scale. This comparison reveals that these host galaxies are not that different. The VLT images of GRB 980425 are considerably deeper and surface brightness dimming will also suppress the fainter structures in the more remote galaxy. One difference is the location of the GRB within the galaxy. GRB 030329 appears to have exploded right on the brightest pixel in the host galaxy. The location is marked by a ring and a cross in Fig. 1. The occurrence of GRB 980425 is instead way outside the center of the galaxy, at a projected distance of about $2.2 \mathrm{kpc}$. This region does not appear to be special in any way, compared for example to the very actively star forming region $850 \mathrm{pc}$ northwest of the explosion site (Fig. 1). We note that at cosmological distances such a spatial difference would not have been possible to resolve.

From Gorosabel et al. (2005) we know that the host of GRB 030329 has a SED that is best fit by a starburst galaxy template. We have also measured the photometry on the HST images and found $\mathrm{m}(\mathrm{F} 435 \mathrm{~W})=23.29, \mathrm{~m}(\mathrm{~F} 606 \mathrm{~W})=23.00$ and $\mathrm{m}(\mathrm{F} 814 \mathrm{~W})=22.83$. These are aperture corrected AB magnitudes. The errors are estimated to be about 0.03 magnitudes. The $\mathrm{B}$ and $\mathrm{V}$ band results are fairly consistent with Gorosabel et al. (2005) although the I band is measured to be fainter in the HST images. Looking also at the spatially resolved photometry, we note that the colors are significantly redder in the outer parts of the galaxy. This means that using an integrated magnitude will overestimate the age of the stellar population

(e.g., Östlin et al., 2001). This would make the ages estimated by Gorosabel et al. (2005) more consistent with the expected ages from very massive GRB progenitors.

\section{Comparisons}

Having established and collected the properties of the host galaxies of GRB 980425 and GRB 030329 we will in this section compare these properties with those of other host galaxies. First, we compile the properties of the third nearby GRB/SN host galaxy, that of GRB 031203, in the same way as we have done for the other two 
host galaxies. Thereafter we compare this small sample of galaxies with a sample of more distant Blue Compact Galaxies.

\subsection{Comparison to the GRB 031203 host}

The third nearby SN/GRB host galaxy, the host of GRB 031203, has been spectroscopically studied in detail by Prochaska et al. (2004).

Using their published reddening corrected emission line fluxes we performed the same kind of emission line analysis as for the other hosts. The resulting metallicities are given in Table 2. We note that our values are somewhat higher than those found by Prochaska et al. $(2004,12+\log (\mathrm{O} / \mathrm{H})=8.0)$. This is probably because the method we have used does not take into account the additional temperature information from the fainter [O III] $4363 \AA$ line. This makes our abundance determinations less secure, and is included here to indicate the uncertainties in the metallicities also for the other two host galaxies.

When it comes to extinction, Prochaska et al. 2004) estimated this from the Balmer decrement to be $E(B-V)=1.17$. Most of this, $\sim 1.04$ mag, is due to extinction in our own galaxy (Schlegel et al., 1998). Hence the internal reddening also in this host galaxy is likely to be modest.

Given this extinction, we take the line fluxes from Prochaska et al. (2004) and use the same method as above to infer

$\mathrm{SFR}_{\mathrm{H} \alpha}=13.2 \mathrm{M}_{\odot} \mathrm{yr}^{-1}$

$\mathrm{SFR}_{[\mathrm{O} \text { II }]}=9.6 \mathrm{M}_{\odot} \mathrm{yr}^{-1}$

The X-ray data (Watson et al., 2004) suggest a massive SFR of at most 24 $\pm 17 \mathrm{M}_{\odot} \mathrm{yr}^{-1}$, corresponding to a total SFR of $\lesssim 150 \pm 110 \mathrm{M}_{\odot} \mathrm{yr}^{-1}$.

The low K-band luminosity $\left(\mathrm{L} \sim \mathrm{L}_{K}^{\star} / 5\right)$ and the extrapolated B-band magnitude (Prochaska et al., 2004) corresponds to $L=0.28 L^{\star}$. This gives a SSFR $=\sim 39 \mathrm{M}_{\odot} \mathrm{yr}^{-1}\left(\mathrm{~L} / \mathrm{L}^{\star}\right)^{-1}$.

For this host galaxy we have limited information about the size and galaxy type. Using an $I$-band image obtained at the Danish $1.54 \mathrm{~m}$ telescope 46 days past explosion (Thomsen et al., 2004) we compared Gaussian fits to the host galaxy with fits of the nearby stars. The galaxy is clearly not a point source, but with a seeing of $\sim 11^{\prime \prime} 0$ it is only marginally resolved. Taking into account the extent of the point spread function this means that the galaxy FWHM is about $1-2 \mathrm{kpc}$ on the sky. This is slightly larger than the very compact GRB 030329 host, but not by much (Fig. 1). A J-band image presented by Gal-Yam et al. (2004) shows that the GRB occurred in the central regions of this galaxy. 


\subsection{Comparison to other galaxies}

The properties of the three SN-GRB host galaxies are summarized in Table 5. The star formation rates given in the table are averages between those obtained for $\mathrm{H} \alpha$ and [O II], when available. With only three firmly established GRB/SN host galaxies any conclusion regarding this population will be tentative, but it seems that their overall properties are consistent with those established for more distant GRB host galaxies; small, sub-luminous, metal-poor blue galaxies (e.g., Fruchter et al., 1999; Le Floc'h et al., 2003; Fynbo et al., 2003; Christensen et al., 2004). The host galaxy of GRB 980425 has, however, not a very low metal abundance - and should not be classified as a starburst galaxy.

Christensen et al. (2004) compared the specific star formation rates (UV to optical colours, since the SFRs were estimated from the near-UV continuum) of GRB hosts to galaxies in the Hubble deep field, and concluded that the GRB hosts have higher SSFR than $95 \%$ of the HDF galaxies. Such a comparison may give the appearance that the GRB hosts have very special properties.

However, the HDF comprises a plethora of galaxies of different types with different distances and selection functions. Given that the hosts under study in the present paper are rather nearby, it would be interesting to compare these with galaxies in the local universe with similar properties.

Although not comprising a very homogeneous class, Blue Compact (dwarf) Galaxies (BCGs) and low luminosity emission line selected galaxies (H II-galaxies) typically have luminosities below $\mathrm{L}^{\star}$, low metallicity (Izotov \& Thuan, 1999), low extinction (Izotov et al., 1997) and active star formation (Kunth \& Östlin, 2000). These are properties often ascribed to GRB hosts and the BGC population may therefore serve as an interesting local comparison sample.

Gil de Paz et al. (2003) present visual and $\mathrm{H} \alpha$ luminosities for a large sample of local BCGs, which we have converted into specific star formation rates. We also added a few luminous BCGs with very active star formation from Östlin et al. (2001) to this sample, with the SFRs recalibrated by the Kennicutt (1998) SFR$\mathrm{H} \alpha$ relation for the sake of homogeneity. In Fig. 2 we compare the star formation rates and luminosities of the combined BCG sample with that of the three SN-GRB hosts of this paper. For this comparison we used the SFRs inferred from $\mathrm{H} \alpha$ only, and since Gil de Paz et al. (2003) do not present internal extinction, we use vales corrected for Galactic extinction only in order to be consistent.

The combined samples cover a range in SSFR from $\sim 1$ to $100 \mathrm{M}_{\odot} \mathrm{yr}^{-1}\left(\mathrm{~L} / \mathrm{L}^{\star}\right)^{-1}$ while the typical BCG has a SSFR close to 10 . While this large range of values confirms that this class of galaxies is not very homogeneous, we see that the SN/GRB host galaxies have similar properties to this class. It is among these galaxies that we find the most active star forming sub-luminous galaxies in the local universe. The 
BCGs with SSFR $<10$ have moderately active star formation and this would also be the location occupied by ordinary dwarf irregulars and disk galaxies, such as the host of GRB 980425. The other two SN/GRB hosts would probably have been classified as actively starforming BCGs if they would have been more nearby.

BCGs are believed to account for a significant amount of the SFR also at higher redshifts. Still, in a magnitude limited sample, such galaxies are in minority. In this respect the results by Gorosabel et al. (2005) and Christensen et al. (2004) can be understood - the majority of the (SN/)GRBs occur in the few percent of the magnitude limited population that has the highest SSFR, both at low and high redshifts, i.e., galaxies with properties similar to BCGs/H II-galaxies (see also Courty et al., 2004).

\section{Conclusions}

We have mined several archives and data sets in order to characterize the host galaxies that are known to have harbored a GRB-SN. The picture of compact low luminosity, metal poorish galaxies in a starforming phase is established, consistent with other studies of ordinary and more distant GRBs (Le Floc'h et al., 2003; Christensen et al., 2004). The local extinction in these galaxies appears to be small. We have proposed that a population of Blue Compact Galaxies have similar properties to the GRB-SN host galaxies.

For the host of GRB 980425 we have for the first time derived the star-formation rate. We have shown that this galaxy is not very metal-poor, and that a population study based on the broad-band photometry is consistent with a normal starforming galaxy with continuous star formation over 5-7 Gyrs, i.e., not with a starburst galaxy. A similar investigation of the spatially resolved H II region where the GRB occurred gives us an estimate of the GRB progenitor mass of $\gtrsim 30 M_{\odot}$. This is consistent with theoretical scenarios of SN $1998 \mathrm{bw}$ being due to a very massive star (e.g., Iwamoto et al., 1998). Today, collapsar models - involving the collapse of massive stars - are the favoured models for long GRBs (e.g., MacFadyen \& Woosley, 1999; Hiorth et al., 2003), and it is therefore very encouraging that this population study confirms such models. Similar observational contraints on the mass of supernova progenitors are becoming very useful for constraining SN models (see e.g., Maund \& Smartt, 2005, and references therein).

For the host of GRB 030329 we provide high-resolution HST imaging which reveals the morphology and location of the burst. It shows the host to be a very compact galaxy indeed. An early high-resolution spectrum provides a very accurate redshift determination and the lack of sodium lines in this spectra supports a very low extinction towards the GRB. The width of the resolved $\mathrm{H} \alpha$ provides an estimate of the dynamical mass of the galaxy. Our low-resolution spectra give useful 
constraints on the metallicity, but we also show that the analysis is less straightforward than previously acknowledged.

This study thus provides a smorgasbord of what can be learned from optical data of relatively nearby host galaxies. We hope that more such galaxies will be discovered with the Swift satellite. To characterize this population is important to understand selection biases in determining the high-z starformation rates via GRB selected galaxies. Swift has the potential to point us to a large number of such distant galaxies.

The datasets used in this investigation were mainly obtained to study the individual SNe-GRBs and were not really optimized for the host studies. This is particularly true for the spectroscopy. Clearly, better observations are possible to obtain when the $\mathrm{SNe}$ have faded away.

Acknowledgements. We want to thank Javier Gorosabel for discussions about several important aspects of this paper. We also want to thank Lisa Kewley for providing her metallicity script. Thanks to Christina Thöne for comments. Part of this research was conducted at the Dark Cosmology Centre funded by The Danish National Research Foundation. Important VLT observations were conducted on ESO Director's Discretionary Time and were obtained by Paranal staff. We are grateful for these efforts.

\section{References}

Allende Prieto, C., Lambert, D. L., \& Asplund, M. 2001, ApJ, 556, L63

Berger, E., Cowie, L. L., Kulkarni, S. R., Frail, D. A., Aussel, H., \& Barger, A. J. 2003, ApJ, 588, 99

Bloom, J. S., Djorgovski, S. G., Kulkarni, S. R., \& Frail, D. A. 1998, ApJ, 507, L25

Bloom, J. S., Kulkarni, S. R., Djorgovski, S. G., et al. 1999, Nature, 401, 453

Bressan, A., Fagotto, F., Bertelli, G., \& Chiosi, C. 1993, A\&AS, 100, 647

Christensen, L., Hjorth, J., \& Gorosabel, J. 2004, A\&A, 425, 913

Courty, S., Björnsson, G., \& Gudmondsson, E. H. 2004, MNRAS, 354, 581

Coleman, G.P., Wu, C.-C., \& Weedman, D.W. 1980, ApJSS, 43, 393

Edmunds, M. G. \& Pagel, B. E. J. 1984, MNRAS, 211, 507

Ellison, S. L., Kewley, L. J., \& Mallén-Ornelas, G. 2005, MNRAS, 357, 354

Fagotto, F., Bressan, A., Bertelli, G., \& Chiosi, C. 1994, A\&AS, 105, 29

Fioc M., \& Rocca-Volmerange B. 1997, A\&A, 326, 950

Fioc M., \& Rocca-Volmerange B. 1999, astro-ph/9912179

Fruchter, A. S., Thorsett, S. E., Metzger, M. R., et al. 1999, ApJ, 519, L13

Fruchter, A. S., Krolik, J. H., \& Rhoads, J. 2001, ApJ, 563, 597

Fynbo, J. P. U., Holland, S., Andersen, M. I., et al. 2000, ApJ, 542, L89

Fynbo, J. P. U., Jakobsson, P., Hjorth, J., et al. 2003, A\&A, 406, L63

Galama, T. J., Vreeswijk, P. M., van Paradijs, J., et al. 1998, Nature, 395, 670 
Galama, T. J. \& Wijers, R. A. M. J. 2001, ApJ, 549, L209

Gal-Yam, A., Moon, D.-S., Fox, D. B., et al. 2004, ApJ, 609, L59

Gil de Paz, A., Madore, B. F., \& Pevunova, O. 2003, ApJS, 147, 29

Gorosabel, J., Christensen, L., Hjorth, J., et al. 2003, A\&A, 400, 127

Gorosabel, J., Perez-Ramirez, D., Sollerman, J., et al. 2005, submitted to A\&A

Greiner, J., Peimbert, M., Estaban, C., et al. 2003, GCN, 2020

Grimm, H.-J., Gilfanov, M., \& Sunyaev, R. 2003, MNRAS, 339, 793

Guzman, R., Gallego, J., Koo, D. C., Phillips, A. C., Lowenthal, J. D., Faber, S. M., Illingworth, G. D., \& Vogt, N. P. 1997, ApJ, 489, 559

Guzmán, R., Östlin, G., Kunth, D., Bershady, M. A., Koo, D. C., \& Pahre, M. A. 2003, ApJ, 586, L45

Hamuy, M., Suntzeff, N. B., Heathcote, S. R., Walker, A. R., Gigoux, P., \& Phillips, M. M. 1994, PASP, 106, 566

Hjorth, J., Sollerman, J., Møller, P., et al. 2003, Nature, 423, 847

Hobbs, L. M. 1974, ApJ, 191, 381

Holmberg, E. B., Lauberts, A., Schuster, H. E., \& West, R. M. 1977, A\&AS, 27, 295

Iwamoto, K., Mazzali, P., Nomoto, K., et al. 1998, Nature, 395, 672

Izotov, Y. I., \& Thuan, T. X. 1999, ApJ, 511, 639

Izotov, Y. I., Thuan, T. X., \& Lipovetsky, V.A. 1997, ApJS, 108, 1

Jakobsson P., Björnsson G., Fynbo J. P. U. et al. 2005, MNRAS, in press (astro-ph/0505542)

Kennicutt, R. C. 1998, ARA\&A, 36, 189

Kewley, L. J., \& Dopita, M. A. 2002, ApJS, 142, 35

Kouveliotou, C., Woosley, S. E., Patel, S. K., et al. 2004, ApJ, 608, 872

Kunth, D., \& Östlin, G. 2000, A\&ARv, 10, 1

Landolt, A. U. 1992, AJ, 104, 340

Lauberts, A., \& Valentijn, E. A. 1989, The surface photometry catalogue of the ESO-Uppsala galaxies, European Southern Observatory

Lee, H., Grebel, E. K., \& Hodge, P. W. 2003, A\&A, 401, 141

Lee, J. C., Salzer, J. J., \& Melbourne, J. 2004, ApJ, 616,752

Le Floc'h, E., Duc, P.-A., Mirabel, I. F., et al. 2003, A\&A, 400, 499

MacFadyen, A. I., \& Woosley, S. E. 1999, ApJ, 524, 262

Malesani, D., Tagliaferri, G., Chincarini, G., et al. 2004, ApJ, 609, L5

Matheson, T., Garnavich, P. M., Stanek, K. Z., et al. 2003, ApJ, 599, 394

Maund, J. R., \& Smartt, S. J. 2005, MNRAS, 360, 288

Meynet, G., Maeder, A., Schaller, G., Schaerer, D., \& Charbonnel, C. 1994, A\&AS, 103, 97

Oke, J. B. 1990, AJ, 99, 1621

Patat, F., Cappellaro, E., Danziger, J., et al. 2001, ApJ, 555, 900

Persic, M., Rephaeli, Y., Braito, V., Cappi, M., Della Ceca, R., Franceschini, A., \& Gruber, D. E. 2004, A\&A 419, 849

Prochaska, J. X., Bloom, J., Chen, H.-W., et al. 2004, ApJ, 611, 200

Salpeter, E. E. 1955, ApJ, 121, 161

Schlegel, D. J., Finkbeiner, D. P., \& Davis, M. 1998, ApJ, 500, 525 
Sollerman, J., Kozma, C., Fransson, C., Leibundgut, B., Lundqvist, P., Ryde, F., \& Woudt, P. 2000, ApJ, 537, L127

Sollerman, J., Holland, S., Challis, P., et al. 2002, A\&A, 386, 944

Sollerman, J., Cox, N., Mattila, S., et al. 2005, A\&A, 429, 559

Stanek, K. Z., Matheson, T., Garnavich, P. M., et al. 2003, ApJ, 591, L17

Thomsen, B., Hjorth, J., Watson, D., et al. 2004, A\&A, 419, L21

Warren, S. J., Møller, P., Fall, S. M., \& Jakobsen, P. 2001, MNRAS, 326, 759

Watson, D., Hjorth, J., Jakobsson, P., Pedersen, K., Patel, S., \& Kouveliotou, C. 2004, A\&A, 425, L33

Waxman, E. \& Draine, B. T. 2000, ApJ, 537, 796

Östlin, G., Amram, P., Bergvall, N., Masegosa, J., Boulesteix, J., \& Márquez, I. 2001, A\&A, 374, 800 

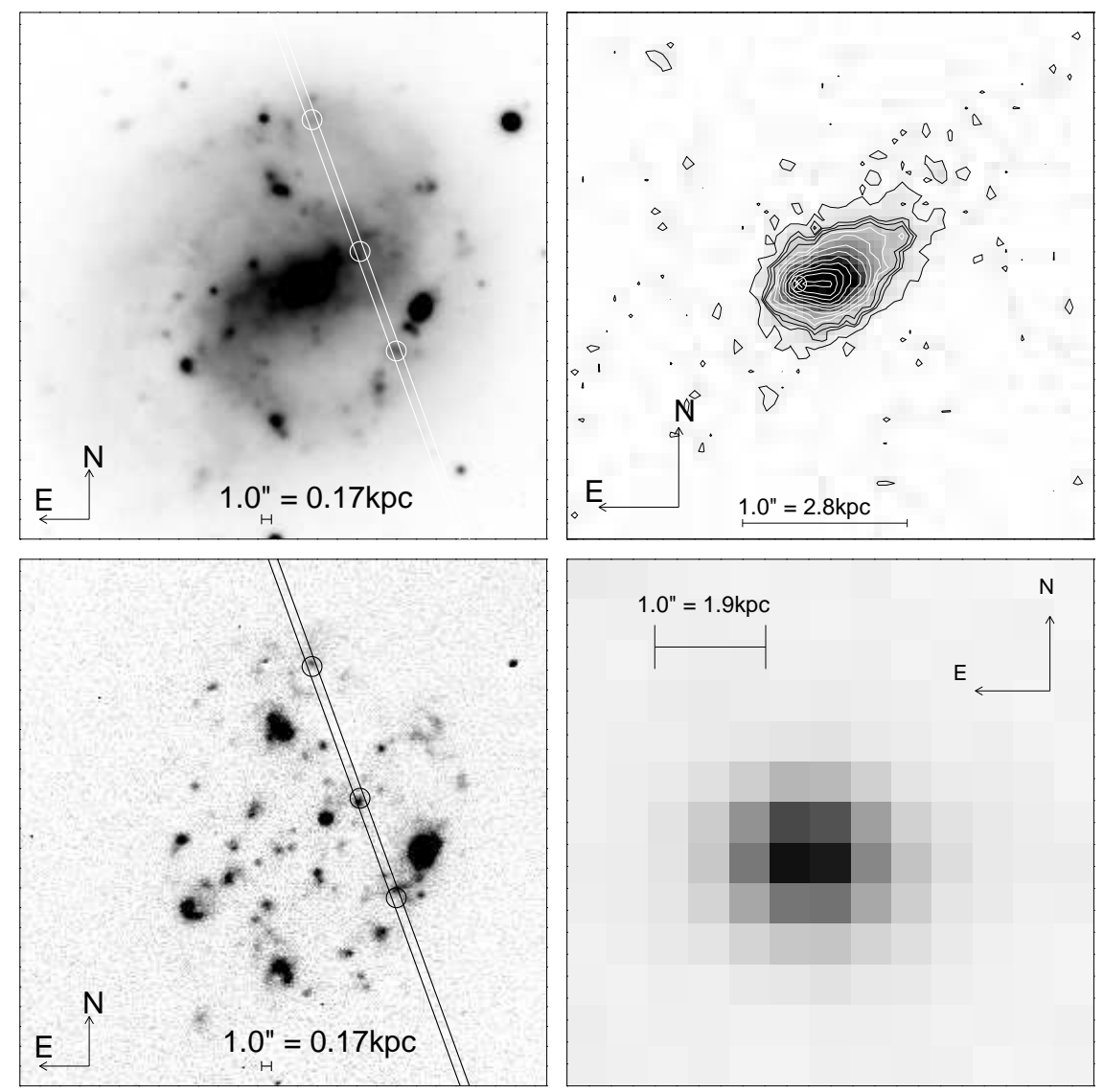

Fig. 1. Upper left: The host galaxy of GRB 980425. This is a V-band image obtained with the VLT. The FOV of this image is 53'. $2 \times 533^{\prime \prime} 2$ which corresponds to $9.3 \mathrm{kpc}$ at the distance of this galaxy. The slit position and orientation is marked by white lines. The three regions investigated are marked. The GRB explosion site is the South-Western of these regions. Upper right: The host galaxy of GRB 030329. This is a F606W image obtained with the HST. The FOV is the same physical $9.3 \mathrm{kpc}$ as for the GRB 980425 host shown to the left. Contours are overlaid the emphasize also the fainter parts of the galaxy. The position of the GRB is marked by a white ring and a cross. Lower left: This is the $\mathrm{H} \alpha$ image of the GRB 980425 host galaxy. Compare to the image above. Lower right: This is an I-band image obtained of the host galaxy for GRB 031203 with the Danish $1.54 \mathrm{~m}$ telescope. The FOV is the same physical $9.3 \mathrm{kpc}$ as for the other host galaxies, but in this case the galaxy is only marginally resolved. 


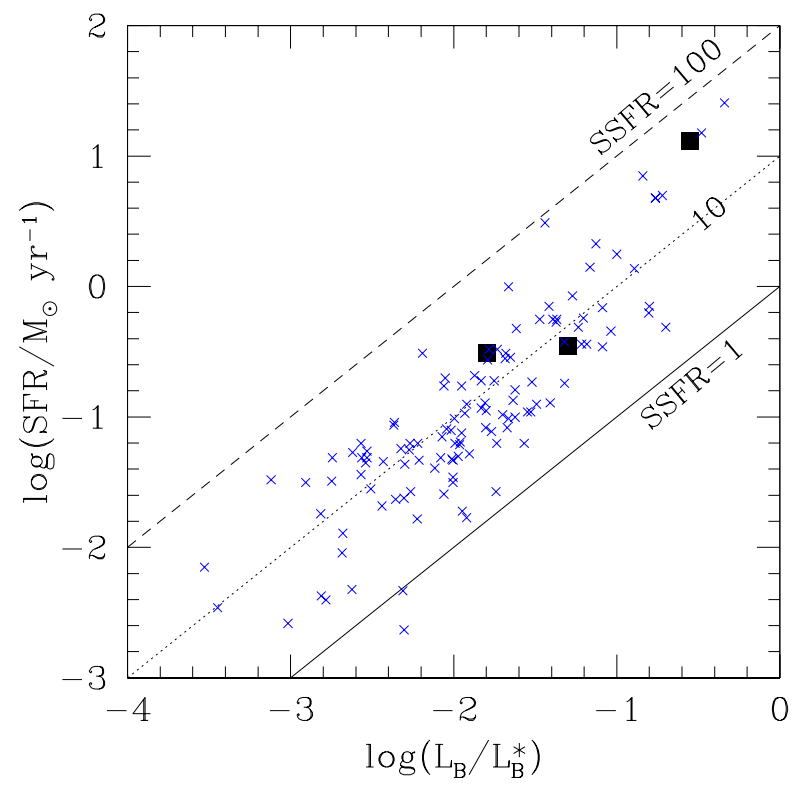

Fig. 2. Star formation rate vs luminosity. The y-axis shows the logarithm of the star formation rate as derived from $\mathrm{H} \alpha$, while the x-axis shows the logarithm of the blue luminosity. The three GRB hosts marked by solid squares are seen to occupy the same region as the sample of local blue compact galaxies indicated by cross-symbols. Lines of constant specific star formation rate (SSFR) are indicated. 
Table 1

Emission line ratios

\begin{tabular}{lccc}
\hline \hline Line $(\AA)$ & $980425^{a}$ & $030329^{b}$ & $031203^{c}$ \\
\hline O II 3727 & 4.54 & 1.61 & 1.06 \\
Ne III 3870 & 0.40 & 0.22 & 0.60 \\
Ne III 3970 & 0.24 & - & 0.23 \\
H $\delta$ 4103 & 0.22 & 0.15 & 0.25 \\
H $\gamma 4342$ & 0.44 & 0.36 & 0.49 \\
H $\beta$ 4863 & 1.0 & 1.0 & 1.0 \\
O III 4960 & 1.07 & 1.12 & 2.11 \\
O III 5008 & 2.77 & 3.40 & 6.36 \\
He I 5877 & 0.15 & - & 0.12 \\
H $\alpha$ 6565 & 2.45 & 2.74 & 2.82 \\
N II 6585 & 0.38 & - & 0.15 \\
S II 6726 & 0.89 & 0.40 & 0.16 \\
\hline
\end{tabular}

${ }^{a}$ Relative to $\mathrm{H} \beta$, Corrected only for Galactic extinction of $E(B-V)=0.059$.

${ }^{b}$ Corrected only for Galactic extinction of $E(B-V)=0.025 \mathrm{mag}$. Total $\mathrm{L}(\mathrm{H} \alpha)=2.7 \times 10^{40} \mathrm{erg} \mathrm{s}^{-1}$.

${ }^{c}$ GRB 031203 from Prochaska et al. (2004), corrected for $E(B-V)=1.17$. Total $\mathrm{L}(\mathrm{H} \alpha)=1.7 \times 10^{42} \mathrm{erg} \mathrm{s}^{-1}$. 
Table 2

Host metallicities

\begin{tabular}{lccc}
\hline \hline Host & Combined $^{a}$ & $\begin{array}{c}\text { R23 Comb } \\
{[\log (\mathrm{O} / \mathrm{H})+12]}\end{array}$ & Comp Ave \\
\hline $031203^{b}$ & 8.19 & 8.21 & 8.18 \\
$030329^{c}$ & 8.65 & 8.65 & 8.67 \\
$980425 \mathrm{SN}$ & 8.62 & 8.43 & 8.56 \\
$980425 \mathrm{C}$ & 8.61 & 8.67 & 8.73 \\
$980425 \mathrm{NE}$ & 8.64 & 8.44 & 8.57 \\
\hline
\end{tabular}

${ }^{a}$ Kewley \& Dopita (2002) provide details for the various methods.

${ }^{b}$ Prochaska et al. (2004) found $7.98 \pm 0.15$ using more lines.

${ }^{c}$ The lower branch value of $\sim 7.9$ can not be excluded.

Table 3

Photometry for ESO 184-G82

\begin{tabular}{|c|c|c|c|c|c|c|c|c|c|}
\hline \multirow{2}{*}{\multicolumn{2}{|c|}{ Region }} & \multirow[t]{2}{*}{$B$} & \multirow[t]{2}{*}{$B-V$} & \multirow[t]{2}{*}{$V-R$} & \multirow[t]{2}{*}{$R-I$} & & & & \\
\hline & & & & & & & & & \\
\hline \multicolumn{2}{|c|}{ Entire galaxy $^{a}$} & 14.94 & 0.40 & 0.40 & 0.41 & & & & \\
\hline \multicolumn{2}{|c|}{ Local H II region } & 22.56 & 0.21 & 0.02 & -0.12 & & & & \\
\hline \multicolumn{10}{|c|}{${ }^{a}$ Magnitude measured down to $25 \mathrm{mag} \operatorname{arcsec}^{-2}$. Corrected for Galactic extinction onl } \\
\hline \multicolumn{9}{|c|}{ Table 4} & Modeling for the local H II region explosion site in ESO-184 \\
\hline \multirow[b]{2}{*}{$\mathrm{Z}$} & \multicolumn{3}{|c|}{$E(B-V)=0.0$} & \multicolumn{3}{|c|}{$E(B-V)=0.05$} & \multicolumn{3}{|c|}{$E(B-V)=0.10$} \\
\hline & $\begin{array}{l}\text { Age } \\
(\mathrm{Myr})\end{array}$ & $\begin{array}{l}\text { RMS } \\
\text { (mag) }\end{array}$ & $\begin{array}{c}\Delta_{\mathrm{EW}} \\
(\AA)\end{array}$ & $\begin{array}{l}\text { Age } \\
(\mathrm{Myr})\end{array}$ & $\begin{array}{l}\text { RMS } \\
\text { (mag) }\end{array}$ & $\begin{array}{l}\Delta_{\mathrm{EW}} \\
(\AA)\end{array}$ & $\begin{array}{l}\text { Age } \\
\text { (Myr) }\end{array}$ & $\begin{array}{l}\text { RMS } \\
\text { (mag) }\end{array}$ & $\begin{array}{l}\Delta_{\mathrm{EW}} \\
(\AA)\end{array}$ \\
\hline 0.020 & 6 & 0.100 & -83 & 6 & 0.061 & -83 & 6 & 0.090 & -83 \\
\hline 0.010 & 6 & 0.082 & -51 & 6 & 0.040 & -51 & 6 & 0.088 & -51 \\
\hline 0.005 & 7 & 0.078 & -44 & 7 & 0.055 & -44 & 7 & 0.102 & -44 \\
\hline
\end{tabular}

Age: best fitting age in Myr, RMS: root mean square deviation of broad band least square fit, $\Delta_{\mathrm{EW}}=\mathrm{EW}(\mathrm{H} \alpha)_{\text {model }}-\mathrm{EW}(\mathrm{H} \alpha)_{\text {observed }}$ in Ångström. 
Table 5

Properties of the GRB/SN host galaxies

\begin{tabular}{lccc}
\hline \hline GRB & 980425 & 030329 & 031203 \\
$\mathrm{SN}$ & $1998 \mathrm{bw}$ & $2003 \mathrm{dh}$ & $20031 \mathrm{w}$ \\
\hline Redshift & 0.0085 & 0.1685 & 0.1055 \\
$\mathrm{~L}_{B} / \mathrm{L}_{B}^{\star}$ & 0.05 & 0.016 & 0.28 \\
$\mathrm{SFR}\left(\mathrm{M}_{\odot} \mathrm{yr}^{-1}\right)$ & 0.35 & 0.4 & 11 \\
$\mathrm{SFR}_{\mathrm{X}}\left(\mathrm{M}_{\odot} \mathrm{yr}^{-1}\right)$ & $2.8 \pm 1.9$ & $\lesssim 200 \pm 80$ & $\lesssim 150 \pm 110$ \\
$\mathrm{SSFR}\left(\mathrm{M}_{\odot} \mathrm{yr}^{-1}\left(\mathrm{~L} / \mathrm{L}^{\star}\right)^{-1}\right)$ & 7 & 25 & 39 \\
$\log [\mathrm{O} / \mathrm{H}]+12$ & 8.6 & $8.6^{a}$ & 8.2 \\
$\mathrm{Size}(\mathrm{kpc})$ & $12 \times 10$ & 3.9 & $\mathrm{a} \mathrm{few}$ \\
$E(B-V)(\mathrm{mag})$ & 0.059 & $0.025-0.2$ & 1.17 \\
Age $(\mathrm{Myr})$ & 4500 & 150 & - \\
\hline
\end{tabular}

${ }^{a}$ Assuming upper branch 